\title{
Desmame Precoce: Falta de Conhecimento ou de Acompanhamento?
}

\author{
Early Weaning: Lack of Knowledge or Lack of Follow Up?
}

\author{
Suzely Adas Saliba MOIMAZ ${ }^{1}$, Orlando SALIBA ${ }^{2}$, Heloisa Carvalho BORGES ${ }^{3}$, \\ Najara Barbosa da ROCHA ${ }^{4}$, Nemre Adas SALIBA ${ }^{2}$
}

${ }^{1}$ Professora Titular do Departamento de Odontologia Preventiva e Social da Faculdade de Odontologia de Araçatuba da Universidade Estadual Paulista (UNESP), Araçatuba/SP, Brasil.

${ }_{2}^{2}$ Professor Titular do Programa de Pós-Graduação em Odontologia Preventiva e Social da Faculdade de Odontologia de Araçatuba da Universidade Estadual Paulista (UNESP), Araçatuba/SP, Brasil.

${ }^{3}$ Mestranda do Programa de Pós-Graduação em Odontologia Preventiva e Social da Faculdade de Odontologia de Araçatuba da Universidade

Estadual Paulista (UNESP), Araçatuba/SP, Brasil.

${ }^{4}$ Doutoranda do Programa de Pós-Graduação em Odontologia Preventiva e Social da Faculdade de Odontologia de Araçatuba da Universidade Estadual Paulista (UNESP), Araçatuba/SP, Brasil.

\section{RESUMO}

Objetivo: Verificar conhecimentos de mulheres e orientações recebidas por elas sobre aleitamento materno, durante a gestação e após o nascimento dos bebês, e as influências destes sobre a prática da amamentação.

Métodos: Trata-se de um estudo longitudinal, com acompanhamento de 84 pares de mães e bebês, da gestação ao sexto mês de vida dos bebês. Na primeira fase da pesquisa foram realizadas entrevistas domiciliares e em Unidades Básicas de Saúde com as gestantes, para obtenção de suas intenções e conhecimentos sobre a prática do aleitamento materno. Na segunda fase foi realizado acompanhamento das mães e bebês, do primeiro ao sexto mês de idade, para registro das dificuldades na amamentação e motivos de desmame.

Resultados: No primeiro mês de vida, $94,3 \%$ (82) dos bebês foram amamentados, destes, apenas $49,4 \%$ (43) receberam leite materno exclusivamente. Ao final do sexto mês, $43,7 \%$ (38) dos bebês já tinham sido desmamados. Nenhuma mãe amamentava exclusivamente seu bebê ao sexto mês de vida. Durante a gravidez, $60,7 \%$ (51) das mães não receberam orientações sobre o aleitamento, e 83,4\% (70) das mães receberam orientações sobre aleitamento após o nascimento das crianças. A maioria das mães $(76,2 \%)$ tinha conhecimento do período ideal de amamentação. Um total de $73(86,9 \%)$ mães acreditava que o aleitamento era benéfico ao bebê, mas somente $41(48,8 \%)$ destas amamentaram. Somente $11(13,1 \%)$ mulheres do estudo foram acompanhadas pela equipe do sistema público de saúde durante a lactação.

Conclusão: Apesar da maioria das gestantes terem sido orientadas e possuírem conhecimento sobre o aleitamento materno, houve desmame precoce. Orientar é importante, entretanto, o apoio e o acompanhamento das mães são fundamentais para que se tenha sucesso na prática da amamentação.

\section{ABSTRACT}

Objective: To assess the knowledge of women and the instructions received on breastfeeding during pregnancy and after childbirth, and their influence on breastfeeding practice.

Method: This study was a longitudinal investigation following 84 pairs of mothers and babies from pregnancy through the sixth month of baby's life. In the first phase of the research, interviews were made with the pregnant women at home and at Basic Health Units to collect information about their intentions and knowledge of breastfeeding. In the second phase of the research, mothers and babies were followed up from the first to sixth month of baby's life to record the difficulties of breastfeeding and reasons for weaning.

Results: In the first month, $94.3 \%$ (82) of the babies were breastfed, but only $49.4 \%$ (43) were breastfed exclusively. At the end of the sixth month of life, $43.7 \%$ (38) of the babies had already been weaned. No mother breastfed exclusively her baby within the sixth month. During pregnancy, $60.7 \%$ (51) of the mothers had no instructions about breastfeeding and $83.4 \%(70)$ of the mothers received instructions on breastfeeding after childbirth. Most mothers (76.2\%) knew about the ideal breastfeeding period. Seventy-three $(86.9 \%)$ mothers believed that breastfeeding was beneficial for the baby, but only $41(48.8 \%)$ of them really breastfed. Only $11(13.1 \%)$ mothers were followed up by the public health system team during lactation.

Conclusion: Although most women had been instructed during pregnancy or after childbirth and had knowledge of breastfeeding, early weaning occurred. Advising is important, but the support and follow up of the mothers are of utmost importance for a successful breastfeeding practice.

\section{DESCRTTORES}

Aleitamento materno; Desmame; Educação em saúde.

\section{KEY-WORDS}

Breastfeeding; Weaning; Health education. 


\section{INTRODUÇÃO}

O leite materno é o alimento ideal para o bebê e tem sido recomendado como alimento exclusivo até os seis primeiros meses de vida. Depois dos seis meses, com o objetivo de suprir suas necessidades nutricionais, a criança deve começar a receber alimentação complementar segura e nutricionalmente adequada, juntamente com a amamentação, até os dois anos de idade $^{1}$. O aleitamento materno (AM) é de extrema importância para a sobrevivência e crescimento do bebê, pois atende todas as necessidades nutricionais, imunológicas e emocionais do recém-nascido ${ }^{2}$. As mães também experimentam vantagens com a amamentação que incluem aceleração da perda de peso ganho na gravidez e da involução uterina pós-parto e menor incidência de câncer de mama e de ovário. $O$ aleitamento também traz benefícios para a família, sendo uma opção econômica e prática ${ }^{3}$.

Desta forma, a promoção do AM deveria ser vista como ação prioritária para a melhoria da saúde e da qualidade de vida das crianças e de suas famílias. Promover o AM pode ser um bom exemplo de política pública que envolve a família, comunidade, governos e sociedade civil, com baixo custo e excelente impacto sobre o desenvolvimento infantil ${ }^{4}$. A partir da década de 1990, várias normatizações e ações foram desencadeadas em nível nacional e internacional, com o objetivo de ampliar a divulgação dos benefícios e a prática do AM. Dentre elas destacam-se a Declaração de Innocenti, o estabelecimento da Norma Brasileira de Comercialização de Alimentos para Lactentes e Crianças de Primeira Infância (NBCAL), a iniciativa Hospital Amigo da Criança (IHAC) e a revigoração dos Programas de Puericultura em Unidades Básicas de Saúde ${ }^{5}$.

Apesar da ampla divulgação nas maternidades, postos de puericultura e campanhas de saúde pública, a prática do AM ainda não atingiu a frequência e duração desejáveis, embora venha evoluindo favoravelmente, porém de maneira muito lenta, durante os últimos 30 anos $^{6,7}$. A prática da amamentação envolve uma complexa interação de fatores socioeconômicos, culturais e psicológicos ${ }^{8}$. Para que a amamentação natural seja uma prática frequente, é necessário avaliar e entender o que as mães pensam em relação ao período da amamentação, e também os motivos por que elas a interrompem ${ }^{5}$. Desafio ainda maior é descobrir quais as ações podem atuar nesses fatores, modificando-os de forma a promover maior adesão das mães à prática do AM.

Os profissionais de saúde são importantes no incentivo do AM, apoiando e instruindo a nutriz, pelo acompanhamento pré-natal cuidadoso, formação de grupos de gestantes, alojamento conjunto, durante a puericultura e na promoção de campanha de incentivo ao aleitamento ${ }^{8}$. Afinal, na medida em que se conhecem os motivos que possam contribuir com o desmame precoce, pode-se atuar melhor no sentido de prevenção desses fatores de forma mais direcionada e, portanto, mais eficaz ${ }^{9}$. Além de conhecer os motivos que levam ao desmame precoce, é fundamental acompanhar e apoiar as mães para que as mesmas se sintam capazes de enfrentar as dificuldades que possam surgir.

Desta forma, é de grande importância avaliar as orientações sobre AM recebidas pelas mães e verificar o quanto essas orientações são capazes de motivá-las, modificando comportamentos e levando à prática do AM. Portanto, esse estudo longitudinal tem como objetivo analisar, no serviço público, o conhecimento das mães e as orientações recebidas por elas sobre AM durante a gestação e lactação, bem como verificar a associação entre esses fatores e a prática do AM.

\section{METODOLOGIA}

\section{Amostra}

A população de estudo constituiu de 101 mulheres que estavam no terceiro trimestre de gestação, cadastradas no serviço público de saúde de dois municípios do Estado de São Paulo, Araçatuba e Piacatu, no período de março a julho de 2007. Todas as gestantes foram visitadas em seus domicílios ou entrevistadas na própria Unidade Básica de Saúde. Foram excluídas do estudo as gestantes que estavam com endereço cadastrado não encontrado, errado, ou incompleto; as que se recusaram a participar do estudo e as que não estavam no seu último trimestre de gravidez. A amostra final foi de 84 mães e 87 bebês, uma vez que três mães tiveram gêmeos.

\section{Desenho do estudo}

Foi realizado um estudo de coorte, prospectivo, parte de um estudo maior que acompanhou gestantes desde o pré-natal (último trimestre de gestação) até os 36 meses de idade dos bebês.

Inicialmente, foi realizado um estudo piloto para validação e adequação do instrumento de coleta de dados, aferição das dificuldades encontradas, capacitação e calibração dos pesquisadores. O processo de capacitação consistiu numa exaustiva análise do questionário a ser aplicado, com discussão das questões mais controversas. Para padronização do exame clínico, todos os examinadores foram previamente calibrados. $\mathrm{O}$ processo de calibração constou de uma etapa teórica, com estudo dos indicadores e de casos clínicos, e de uma etapa prática, que incluiu realização de exames e discussão pela técnica do consenso. O valor do teste Kappa, teste de concordância entre os pesquisadores, foi de $\mathrm{K}=0.91$.

O estudo foi realizado em duas fases. A primeira consistiu do acompanhamento das gestantes até a proximidade do parto, e foram avaliadas as condições socioeconômico-cultural e os conhecimentos sobre o AM. Foram realizadas entrevistas domiciliares pelos pesquisadores, previamente calibrados, utilizando um 
formulário semiestruturado, contendo variáveis quantiqualitativas, como dados pessoais da mãe, informações sobre moradia e renda, escolaridade e conhecimento sobre o AM. A segunda fase do estudo foi conduzida após o nascimento dos bebês e envolveu o acompanhamento das mães e de seus bebês, por meio de visitas domiciliares mensais, do primeiro ao sexto mês de vida dos bebês. Durante as visitas mensais, um roteiro foi utilizado para registro das dificuldades em relação ao aleitamento, motivos alegados para a introdução de outros alimentos diferentes do leite materno, e motivos de desmame.

A classificação proposta pela Organização Mundial da Saúde (OMS) foi utilizada para a definição do padrão de aleitamento: aleitamento exclusivo quando a criança consome apenas o leite materno, aleitamento predominante quando além do leite materno há adição de outros líquidos, aleitamento complementar para bebês que consomem outros alimentos sólidos e o leite materno, e por fim, aleitamento artificial quando ocorre o desmame. $\mathrm{O}$ aleitamento total incluiu todas as crianças que foram amamentadas, independentemente de ser de forma exclusiva, predominante ou complementar ${ }^{10}$.

Os dados obtidos foram analisados por meio do programa Epi Info $2000^{11}$, programa para Windows de livre distribuição pela OMS, e o pacote estatístico Bioestat $5.0^{12}$. Os dados foram expressos em média \pm desvio-padrão e porcentagem. Foram utilizadas as seguintes variáveis independentes: conhecimento da mãe sobre a existência de benefício da AM para o filho; conhecimento da mãe sobre a existência de benefício da AM para ela mesma; orientações sobre AM recebidas durante a gestação; orientações sobre AM recebidas após o nascimento dos bebês; acompanhamento das mães pelos serviços de saúde durante o AM. O AM foi utilizado como desfecho. O teste do Qui-quadrado e o Exato de Fisher foram empregados para investigar associações entre as variáveis independentes e o AM. A análise dos períodos de AM exclusivo e AM total foi realizada utilizando-se análise de sobrevivência. Para comparar duas ou mais amostras, dispostas em $\mathrm{n}$ tabelas de contingência e cálculo do odds ratio e do intervalo de confiança (95\%), foi aplicado o teste de sobrevivência de Mantel-Haenzel. Para a análise qualitativa, as respostas foram transcritas na íntegra como foram respondidas e analisadas segundo a "Análise do Conteúdo" preconizada por Bardin ${ }^{13}$. Houve a categorização das respostas e análise em profundidade dos conteúdos.

\section{Aspectos éticos}

Este estudo foi aprovado pelo Comitê de Ética e Pesquisa em Seres Humanos da Faculdade de Odontologia de Araçatuba - FOA/Unesp, sob processo 2006-01471. Todas as mães entrevistadas receberam informações sobre os objetivos do estudo. As mães foram orientadas de que a sua não-participação não lhe acarretaria prejuízo e que os dados coletados permaneceriam anônimos.

\section{RESULTADOS}

As características das 84 mães selecionadas para o estudo estão apresentadas na Tabela 1. A maioria da população do estudo caracterizou-se por ser jovem (25 \pm 5.70 anos), de baixa escolaridade e baixo poder aquisitivo, de cor parda ou negra, viver com os parceiros, ser do lar e não estar na primeira gestação (Tabela 1). Vale ressaltar que dentre as 84 mães do estudo, três delas tiveram gêmeos, portanto, no texto, a descrição de resultados refere-se a 87 bebês.

A taxa de crianças amamentadas no primeiro mês, seguinte ao nascimento, foi de 94,3\% (82) dos bebês, entretanto apenas $43(49,4 \%)$ dos bebês recebiam AM exclusivo durante esse período. No terceiro mês, a taxa de aleitamento exclusivo diminuiu para $13,8 \%$ (12) e nenhum bebê continuou a ser amamentado exclusivamente até o sexto mês de vida. Ao final do sexto mês, quase metade dos bebês já tinham sido completamente desmamados. As curvas de AM exclusivo e AM total estão mostradas na Figura 1.

Maior número de mães recebeu orientações sobre a amamentação, pela equipe de saúde local, após os bebês terem nascidos $(83,4 \%)$ do que durante o período gestacional (39,3\%). As orientações foram transmitidas principalmente pelos médicos $(68,5 \%)$ durante a gravidez e pelas enfermeiras $(77,2 \%)$ após o nascimento dos bebês.

Um total de 64 (76,2\%) mães sabia o tempo ideal que deveriam amamentar exclusivamente, mas apenas 34 (40,5\%) amamentaram exclusivamente durante esse período. Na Tabela 2 é mostrado o conhecimento das mães sobre os benefícios para o bebê e para as mães em se realizar o AM. Havia 73 (86,9\%) mães que acreditavam que existiam benefícios aos bebês, mas apenas $41(48,8 \%)$ amamentaram. Apenas 46 $(54,7 \%)$ mães acreditavam que existiam benefícios para as mães em amamentar e somente 25 (29,8\%) amamentaram (Tabela 2).

Da totalidade de mulheres, 41 (48,8\%) receberam ajuda de algum membro do sistema local de saúde sobre como realizar a amamentação. Na análise qualitativa da opinião dessas mulheres sobre a ajuda recebida, a maioria (78\%) considerou "ótima" ou "boa". Somente $11(13,0 \%)$ mulheres foram acompanhadas pela equipe local de saúde durante a lactação.

Dezessete (20,2\%) mães apresentaram dificuldades em amamentar seu bebê. As principais dificuldades relatadas pelas mães durante a amamentação foram: o bebê não sugava ou não pegava o peito $(10,5 \%)$; problemas no bico do seio como rachaduras, sangramentos, mamas invertidas e falta de bico $(63,1 \%)$; dificuldades em amamentar $(15,8 \%)$; falta de leite ou leite secou $(10,5 \%)$. Observou-se uma relação significante muito forte entre mulheres que tiveram dificuldades em amamentar e a prática do AM $(p<0,0001)$. 
Tabela 1. Características gerais da população de estudo, constituído de mães $(n=84)$ e bebês ( $n=87)$ em dois municípios paulista, Araçatuba e Piacatu, 2010.

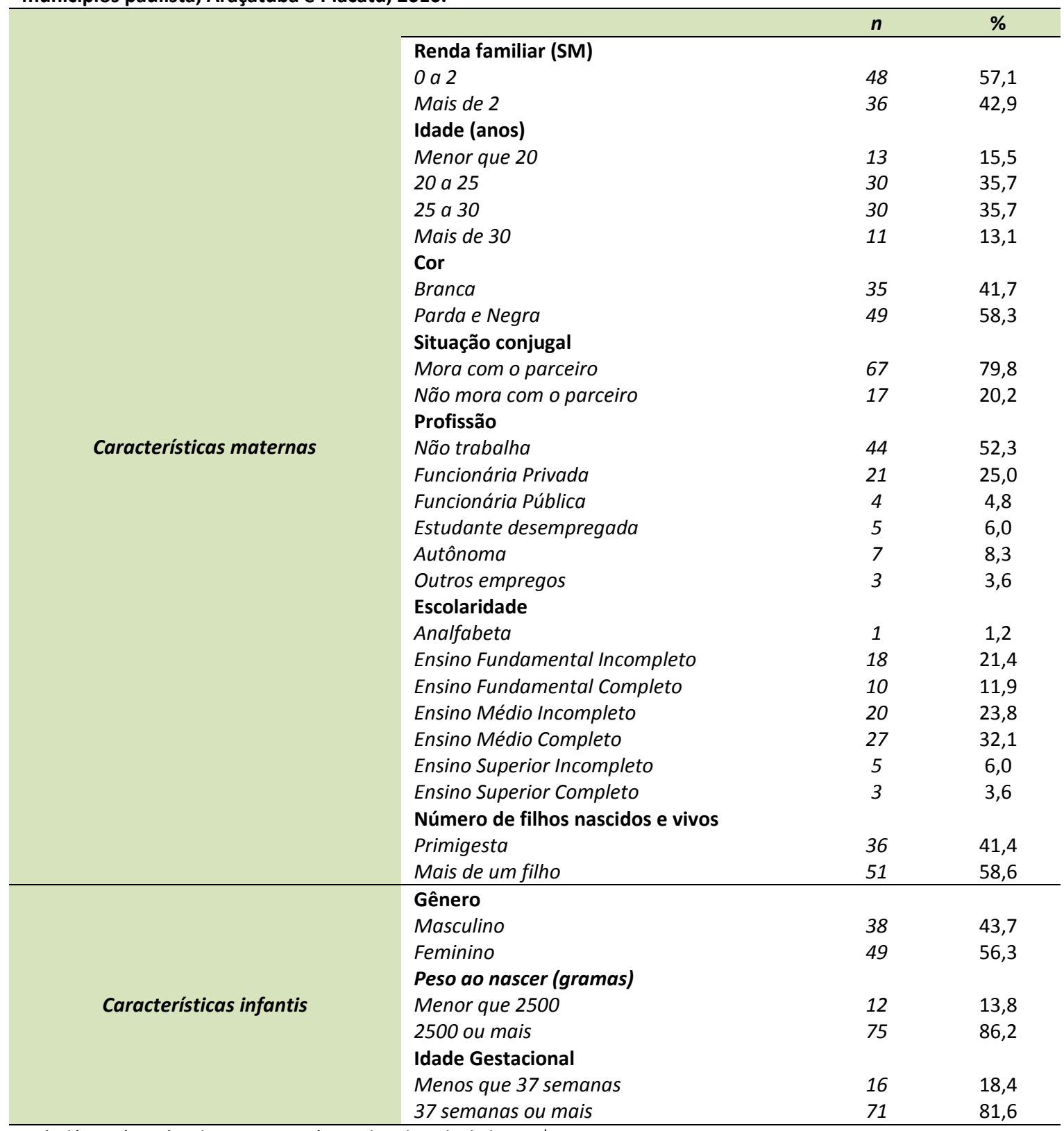

SM (Salário Mínimo): valor vigente na época da coleta de dados - $\mathrm{R} \$ \mathrm{415}, 00$ Fonte: IBGE

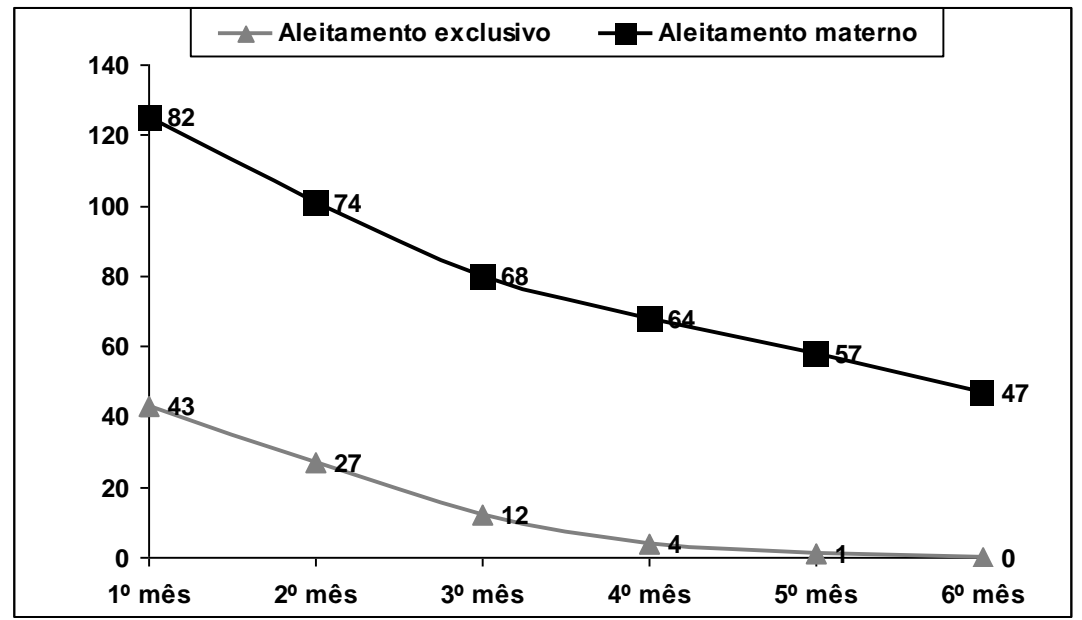

Figura 1. Distribuição numérica das crianças ( $n=87$ ), segundo o aleitamento materno exclusivo e materno total de dois municípios paulista, Araçatuba e Piacatu, 2010. 
Tabela 2. Distribuição numérica de mães segundo seu conhecimento sobre a existência de benefícios do aleitamento materno para o bebê e para a mãe, Araçatuba e Piacatu, 2010.

\begin{tabular}{|c|c|c|c|c|}
\hline \multirow{2}{*}{ Amamentou } & \multicolumn{2}{|c|}{ Existe benefício para o filho } & \multicolumn{2}{|c|}{ Existem benefícios para a mãe } \\
\hline & Sim & Não & Sim & Não \\
\hline Sim & 41 & 6 & 25 & 22 \\
\hline Não & 32 & 5 & 21 & 16 \\
\hline Total & 73 & 11 & 46 & 38 \\
\hline $\begin{array}{c}\text { Valor de } p \\
\text { (Teste Exato de Fisher) }\end{array}$ & \multicolumn{2}{|c|}{1,0000} & \multicolumn{2}{|c|}{0,8286} \\
\hline
\end{tabular}

Tabela 3. Análise dos fatores maternos associados com o aleitamento materno entre 1 a 6 meses, pelo método mantel-Haenzel, Araçatuba e Piacatu, 2010.

\begin{tabular}{lccc}
\hline \multirow{2}{*}{ Variáveis } & \multicolumn{3}{c}{ Aleitamento Materno } \\
\cline { 2 - 4 } & $\mathbf{p}$ & Odds Ratio & IC95\% \\
\hline Está sendo acompanhada pelos serviços de saúde & 0,0543 & 1,8459 & $1,0305-3,3066$ \\
Orientação durante a gestação & 0,0118 & 1,7326 & $1,1454-2,6207$ \\
Orientação após o nascimento & 0,0033 & 2,7737 & $1,4221-5,4099$ \\
\hline
\end{tabular}

As mães que receberam orientações sobre o AM durante a gestação e após o nascimento tiveram uma chance significativamente maior de realizar o AM (odds ratio: 1,7326, IC95\%: 1,1454 - 2,6207; odds ratio: 2,7737, IC95\%: 1,4221 - 5,4099, respectivamente). As mães que eram acompanhadas pelos serviços de saúde tiveram maior tendência de realizar o AM (odds ratio: 1,8459, IC95\%: 1,0305 - 3,3066) (Tabela 3).

\section{DISCUSSÃ̄o}

Apesar de registros recentes na literatura científica de aumento dos índices de aleitamento ${ }^{4,8}$, as condições ideais ainda estão distantes de serem alcançadas e justificam estudos como este, que resgatam medidas de baixo custo na promoção da prática da amamentação. Tais medidas consideram a importância dos profissionais de saúde fornecerem orientações sobre AM às gestantes, nas fases pré e pós-natais e acompanhá-las durante a lactação ${ }^{14,15}$. Atividades de promoção, apoio e proteção ao AM representam importantes estratégias para redução da morbidade infantil, especialmente em países em desenvolvimento ${ }^{16,17}$.

No presente estudo observa-se que as orientações recebidas pelas gestantes sobre a amamentação, durante a gestação e após o nascimento dos bebês, tiveram influência positiva, estatisticamente significante, no padrão de aleitamento. Esses resultados mostram que essas orientações podem ser consideradas como fatores motivadores para as mães em amamentar. As orientações sobre a amamentação são importantes para motivar as mães a iniciarem e manterem o AM. São também cruciais para prover informações sobre as vantagens para as mães e para os bebês em amamentar e para estimularem discussões sobre mitos e tabus relacionados ao tema. Adicionalmente, atividades interativas, como de grupos de gestantes, podem ser utilizadas para ajudar as mulheres aprenderem sobre a experiência de outras mães e sobre o significado do aleitamento. Orientar é importante, porém mais importante que isso é acompanhar as mães no período da lactação, identificando precocemente dificuldades que podem vir a surgir como traumas mamilares, sangramentos, mamadas pouco eficientes. Se esses problemas não forem bem acompanhados e assistidos podem levar ao desmame precoce.

A equipe médica foi a maior responsável pelas orientações às mães durante a gestação, e a enfermeira após o nascimento do bebê, daí a importância de se ter uma equipe treinada e voltada ao estímulo do AM. Os profissionais da saúde, por meio de suas atitudes e práticas, podem influenciar positiva ou negativamente o início da amamentação e sua duração ${ }^{18}$. Em particular, os obstetras, durante o período pré-natal, e os pediatras e a equipe de enfermagem, no período neonatal, podem incentivar a amamentação e apoiar as mães, ajudando-as a iniciá-la precocemente e a adquirirem autoconfiança em sua capacidade de amamentar ${ }^{19,20}$. O desenvolvimento ostensivo de ações de apoio à amamentação dentro das instituições hospitalares que assistem ao parto e ao recém-nascido é reconhecido como medida capaz de modificar o perfil de AM em uma população ${ }^{21,22}$.

O Sistema Público de Saúde Brasileiro possui como base a Estratégia de Saúde da Família, que se constitui num modelo de atenção integral à saúde, com ênfase na promoção e prevenção. A Estratégia de Saúde da Família inclui a formação de equipes multidisciplinares, que são responsáveis pela atenção básica, sendo uma de suas importantes ações a realização de visitas domiciliares em áreas pré-definidas. Nessas visitas, os profissionais de cada equipe realizam atividades de diagnóstico de risco, encaminhamento para tratamento, ações de prevenção e controle de doenças 
crônicas. As equipes de saúde da família ostentam uma condição privilegiada para atividades educativas. A inserção na comunidade, com o envolvimento de lideranças locais, representadas pelos agentes comunitários de saúde, propiciam maiores chances de êxito para atividades educativas ${ }^{4}$. Estudos prévios mostram que, embora esses profissionais possuam informações sobre as vantagens do $A M$, eles não dispõem de informações para manejo das principais dificuldades enfrentadas pelas nutrizes como ingurgitamentos, técnica inadequada, ou problemas com os mamilos. Como constatado em estudos anteriores ${ }^{3}$, um profissional de saúde com treinamento específico, altamente motivado para o incentivo ao AM pode obter melhoras no índice de AM de uma população ${ }^{23,24}$.

No presente estudo, mesmo quando as orientações foram fornecidas, observou-se que os índices de aleitamento estavam abaixo do ideal, uma vez que apenas pouco mais da metade das crianças estavam sendo amamentadas até o sexto mês de vida e nenhuma na forma exclusiva. Sabe-se que o AM exclusivo é a forma mais eficaz e segura de se alimentar o recémnascido. O contato precoce do neonato com proteína de leite de outra espécie constitui significativo fator de risco para doenças alérgicas e autoimunes, tais como bronquites, dermatite atópica, intolerância ou alergia ao leite de vaca e diabetes tipo I. O uso de outros líquidos, rompendo o aleitamento exclusivo, eleva o risco de morbidade, especialmente diarreia, pneumonia e otites $^{25}$

Um aspecto importante a ser considerado nesse estudo é o fato das mães que tiveram dificuldades em amamentar, pela falta de conhecimento e acompanhamento, fracassaram em amamentar seus filhos. Nossos resultados demonstram uma relação significante entre mulheres que tiveram dificuldades de amamentar e a prática do AM $(p<0,0001)$, resultando assim em desmame precoce. Corroborando este trabalho, estudo anterior avaliou, mediante a aplicação de um protocolo preconizado pela Unicef, as necessidades especiais de apoio para um início bem sucedido do AM. Os autores verificaram que diversas dificuldades iniciais, tais como má posição corporal da mãe e do bebê durante a mamada, inadequação da interação mãe/neonato, problemas entre o binômio referentes, principalmente, à amamentação que dificulta a pega adequada e que pode causar traumas mamilares, mamadas pouco eficientes e o não-esvaziamento completo da mama trouxeram consequências negativas para a produção de leite e para o crescimento de bebê, sugerindo que dificuldades no início da amamentação podem conduzir ao desmame precoce ${ }^{26}$.

No presente estudo, apenas $13,0 \%$ das mães receberam acompanhamento da equipe de saúde local durante a lactação. Índice esse muito baixo, sabendo-se da necessidade de se apoiar e acompanhar as mães durante a lactação para que as mesmas se mantenham seguras em suas habilidades e conhecimentos para a contínua realização do AM. Uma vez que poucas mães receberam acompanhamento, esta falta de ajuda e apoio pode ser considerada o motivo de um grande número de mães ter fracassado na prática da amamentação.

Dada a importância que os profissionais de saúde ocupam no processo da amamentação, as equipes multiprofissionais da rede pública deveriam ser estimuladas a ter uma formação mais ampla e continuada sobre AM. Isso poderia ser uma diretriz de política de saúde de baixo custo e certamente de alta resolubilidade, gerando grandes avanços na saúde coletiva, com melhorias no índice de aleitamento ${ }^{27}$.

Uma limitação desse estudo foi as perdas da amostra pela mudança de endereço das gestantes, mudanças de cidade e desistência. Entretanto, considerando que ainda permaneceu um bom número amostral, acreditamos que os resultados desse estudo não foram significativamente comprometidos. Outra limitação foi que avaliamos somente gestantes da rede pública, portanto esses resultados não podem ser extrapolados para gestantes atendidas na medicina suplementar.

\section{CONCLUSÃO}

A educação em saúde, durante a gestação e após o nascimento dos bebês, é importante para a promoção de AM. Porém, somente a educação não foi suficiente para evitar o desmame precoce. 0 acompanhamento das mães durante a lactação é fundamental, mostrando a importância de não só informar, mas também apoiar e acompanhar essas mulheres para que se tenha sucesso na prática do AM.

\section{AGRADECIMENTOS}

Os autores agradecem a Fundação de Amparo à Pesquisa do Estado de São Paulo-Fapesp, pelo auxílio financeiro.

\section{REFERÊNCIAS}

1. World Health Organization (SW). WHA Resolution 54.2. Geneva (SW): World Health Organization; 2001.

2. Popkin BM, Adair JS, Black R. Breast-feeding and diarrheal morbidity. Pediatrics 1990; 86(6):874-82.

3. Giugliani ERJ. Amamentação: como e porque promover? J Pediatr 1994; 70(3):128-47.

4. Parada CMGL, Carvalhaes MABL, Winckler CC, Winckler LA, Winckler VC. Situação do aleitamento materno em população assistida pelo programa de saúde da família-PSF. Rev Latino-am Enfermagem 2005; 13(3):407-14.

5. Del Ciampo LA, Ferraz IS, Daneluzzi JC, Ricco RG, Martinelli CE. Aleitamento materno exclusivo: do discurso à prática. Pediatria 2008; 30(1):22-6.

6. Organização Mundial da Saúde. Proteção, promoção e apoio ao aleitamento materno. Genebra: OMS; 1989. 32p.

7. Braun MLG, Giugliani ERJ, Soares MEM, Giugliani C, Oliveira $A P$, Danelon CMM. Evaluation of the impact of the baby- 
friendly hospital initiative on rates of breastfeeding. Am J Public Health 2003; 93(8):1277-9.

8. Caldeira AP, Fagundes GC, Aguiar GN. Intervenção educacional em eqipes do programa de saúde da família para promoção da amamentação. Rev Saúde Pública 2008; 42(6):1027-33.

9. Losch M, Dungy $\mathrm{Cl}$, Russell $\mathrm{D}$, Dusdieker LB. Impact of attitudes on maternal decisions regarding infant feeding. J Pediatr 1995; 126(4):507-14.

10. World Health Organization. Indicators for assessing breastfeeding practices. Geneva: WHO;2007. Disponível em: http://www.who.int/nutrition/publications/iycf_indicators_for _peer_review.pdf

11. Program Epi Info ${ }^{\mathrm{TM}}$ Version 3.5.1. [citado em 11 Nov 2008] Disponível em: http://www.cdc.gov/epiinfo/

12. Programa BioEstat 5.0 [citado em 11 Nov 2008] Disponível em:

http://www.mamiraua.org.br/download/index.php?dirpath=./ BioEstat\%205\%20Portugues\&order=0

13. Bardin. Análise de conteúdo. 3. ed. São Paulo: Ed. 70, 2004.

14. Chapman DJ. Does maternal diet contribute to colic among breastfed infants? J Hum Lact 2006; 22(2):236-7.

15. Giuliani NR, Oliveira J, Santos BZ, Bosco VL. Prevalência do início do desmame precoce em duas populações assistidas por serviços de puericultura de Florianópolis, SC, Brasil. Pesq Bras Odontoped Clin Integr 2011; 11(2):239-44.

16. Theobald HE. Eating for pregnancy and breast-feeding. J Fam Health Care 2007; 17(2):45-9.

17. Ramalho RA, Saunders C. O papel da educação nutricional no combate às carências nutricionais. Rev Nutr 2000; 13:11-6.

18. Giuliani NR, Oliveira J, Santos BZ, Bosco VL. O início do desmame precoce: motivos das mães assistidas por serviços de puericultura de Florianópolis/SC para esta prática. Pesq Bras Odontoped Clin Integr 2012; 12(1):53-8.

19. Winikoof B, Baer EC. The obstetrician's opportunity: translating "breast is best" from theory to practice. Am J Obstet Gynecol 1980; 138(1):105-17.

20. Patton CB, Beaman M, Sarn C, Lewinski C. Nurses' attitudes and behaviors that promote breast-feeding. J Hum Lact 1996; 12(2):111-5.

21. World Health Organization. World Health Assembly 47.5 Geneva: WHO; 1994.

22. Aldair LS, Popkin BM, Guilkey DK. The duration of breastfeeding: how is it affected by biological, sociodemographic, health sector, and food industry factors? Demography 1993; 30(1):63-80

23. Santiago LB, Bettiol H, Barbieri MA, Guttierrez MRP, Del Ciampo LA. Incentivo ao aleitamento materno: a importância do pediatra com treinamento específico. Arch Pediatr Urug 2005; 76(1):75-84.

24. Giuliani NR, Oliveira J, Traebert J, Santos BZ, Bosco VL. Fatores associados ao desmame precoce em mães assistidas por serviços de puericultura de Florianópolis/SC. Pesq Bras Odontop Clin Integr 2011; 11(3):417-23

25. Victoria C, Smith PG, Vaughan JP, Nobre LC, Lombardi C, Teixeira AM, et AL. Evidence for protection by breastfeeding against infant death from infection disease in Brazil. Lancet 1987; 2(8554):319-22.

26. Carvalhares MABL, Corrêa CRH. Identificação de dificuldades no início do aleitamento materno mediante aplicação de protocolo. J Ped 2003; 79(1):13-20.

27. Ministério da Saúde. Prevalência de aleitamento materno nas capitais brasileiras e no Distrito Federal: relatório preliminar. Brasília; 2001. Disponível:URL: http://tabnet.datasus.gov.br/ cgi/idb2001/d20.pdf. Acessado: 07 de junho de 2003.
Recebido/Received: 29/03/2012

Revisado/Reviewed: 15/12/2012

Aprovado/Approved: 11/02/2013

Correspondência:

Heloisa Carvalho Borges

Rua José Bonifácio, no1193, Vila Mendonça

Programa de Pós-Graduação em Odontologia

Preventiva e Social

CEP: $16015-050$ - Araçatuba-SP

Telefone: (18) 3636-3249/3636-3250

E-mail: heloborges@hotmail.com 